\title{
Towards the quantification of the semantic information encoded in written language
}

\author{
Marcelo A. Montemurro \\ Faculty of Life Sciences, The University of Manchester, M13 9PT, Manchester, \\ United Kingdom \\ m.montemurro@manchester.ac.uk \\ Damián Zanette \\ Consejo Nacional de Investigaciones Cientificas y Técnicas \\ Centro Atómico Bariloche and Instituto Balseiro, 8400 San Carlos de Bariloche, Río Negro, Argentina \\ zanette@cab.cnea.gov.ar
}

\begin{abstract}
Written language is a complex communication signal capable of conveying information encoded in the form of ordered sequences of words. Beyond the local order ruled by grammar, semantic and thematic structures affect long-range patterns in word usage. Here, we show that a direct application of information theory quantifies the relationship between the statistical distribution of words and the semantic content of the text. We show that there is a characteristic scale, roughly around a few thousand words, which establishes the typical size of the most informative segments in written language. Moreover, we find that the words whose contributions to the overall information is larger, are the ones more closely associated with the main subjects and topics of the text. This scenario can be explained by a model of word usage that assumes that words are distributed along the text in domains of a characteristic size where their frequency is higher than elsewhere. Our conclusions are based on the analysis of a large database of written language, diverse in subjects and styles, and thus are likely to be applicable to general language sequences encoding complex information.
\end{abstract}

\section{Introduction}

Among the higher functions of our brain, language stands as a unique ability conferring us a distinctive trait from the rest of living beings [8, 15. Language has developed following the laws of natural evolution [14, 18, 19] with the functional goal of encoding and transmitting information between humans. Although the information to be encoded is usually highly complex, it can be readily projected onto a string of words. It has been argued that this is possible due to the presence of long-range memory in word sequences, which in turn creates organisational structures that go beyond the scope of sentences and paragraphs, and can extend for hundreds or thousands of words [1]. Although, the suggestion of statistical macro-structures associated with the semantic content of linguistic communication has been suggested before [13], it has not yet been accounted for with the use of information theory. 
In recent years the use of tools drawn from statistical physics has quantitatively revealed rich linguistic structures at many scales, ranging from the domain of syntax to the organisation of whole lexicons and literary corpora [5, 4, 6, 9, 10, 16, 24, 25]. However, a fundamental question that has not directly been addressed so far is how statistical structures relate to the function of encoding complex information. The earliest attempt to address this question was probably due to Claude Shannon, soon after the inception of information theory [22]. He represented language as a communication channel encoding information in the sequence of its basic symbols, which in his analysis were 26 letters and the blank space, and obtained an estimate of the entropy of written English [23. However, his analysis was not designed to relate an information measure to the semantic function of language. Instead, it was aimed at reflecting the statistical structure of linguistic sequences independently of the specific information that was being encoded. More recently, we showed that an entropy measure of the word distribution over a text bears information about the specific linguistic role of some word classes [17. This approach disclosed that, for instance, adverbs and most verbs tended to be more uniformly distributed than nouns or pronouns. Thus, simply by associating the entropy measure with a word, it was possible to know whether that word was, for example, more likely an adverb or a noun.

Here, we take a significant step further and propose a measure, based on Shannon's mutual information 22], that captures the relationship between the statistical structure of word sequences and their semantic content. First, we show that words typically appear distributed in domains, so that in certain sections of the text the frequency of a word is consistently higher than elsewhere. This structured heterogeneity in the distribution of words encodes information about the sections of the text in which the words appear. We then use information theory to quantify the relationship between the distribution of words and the sections in a given partition of the text. We find that there is a typical size of the parts for which the information in word distribution is maximal, thus revealing a characteristic scale that can be shown to be related to the semantic content of language. This scale is built up from contributions of all the different individual words, as a direct consequence of the domain structure in their distributions. Finally, we show that the words that contribute the most to the total information are those more closely related to the main topics and subjects if the text.

\section{Domain structure in word distribution}

Although it is evident that different words appear with variable frequency in different parts or sections of a text, it has only recently been pointed out that the patterns of frequency variability are related to the linguistic role of words [17. Additionally, by analyzing the statistics of the distance between consecutive occurrences of the same word in a text, it was shown that many words exhibit a phenomenon of clustering or "self-attraction" [12, 20. Below, we quantify these results within the framework of information theory and show that the long-range distribution of words in written language bears the imprint of the semantic content encoded in the text. First, however, we show that the variability in the frequency of word usage is characterized, for many 
words, by the presence of a series of domains where the frequency of use of the word is higher than outside the domains. Moreover, these domains have a typical size that depends on the specific word. As we shall see below, they contribute to determining the scale of the most informative structures in written language.

In a given text of $N$ words in length, we denote the position of every word by the variable $t=1, \ldots, N$. Then, we can represent the occurrences of a particular word $w$ by a sequence of sharply peaked functions located at the positions where the word $w$ appears. A local average of this sequence, defined as a convolution with a narrow bell-shaped function, allows us to define a rate of occurrence of word $w$ (see Appendix A).

The left panels in Fig. 1 show the rate of occurrence of three words from The Origin of the Species, by Charles Darwin. Panel A shows for the word plants. With its 335 occurrences, this word is considerably frequent in The Origin of Species, but its use is strongly heterogeneous. It tends to appear in localized sections of the text, exhibiting higher frequencies within a number of domains spread throughout the text. The second word we analysed is instinct, shown in panel B. This word appears 69 times, with strongly localized occurrences limited to essentially two sections of the text. The region where it occurs with the highest frequency spans approximately 104 words. The third word, shown in panel C, is for. This is a common English word, appearing 1123 times in the text. Its usage is not directly linked to any specific thematic context. Therefore, apart from seemingly random fluctuations, the rate at which for is used is roughly uniform.

The presence of domains in the distribution of words can be quantitatively revealed by computing the normalized autocorrelation of the rate of occurrence, defined as

$$
c_{w}(\tau)=\frac{\left\langle\rho_{w}(t) \rho_{w}(t+\tau)\right\rangle_{t}-\left\langle\rho_{w}(t)\right\rangle_{t}\left\langle\rho_{w}(t+\tau)\right\rangle_{t}}{\left\langle\rho_{w}(t)^{2}\right\rangle_{t}-\left\langle\rho_{w}(t)\right\rangle_{t}^{2}}
$$

where $\langle\ldots\rangle_{t}$ indicates an average taken over all text positions. If the occurrence of a particular word $w$ is concentrated in localized domains, the autocorrelation function will be significantly different from zero up to a value of $\tau$ of the order of the typical domain size [3]. Panels D, E, and F show the autocorrelation computed for the words of the left-hand panels (black curve). We also show the autocorrelation obtained for the same words after all the words in the text have been randomly shuffled, thus destroying any pattern in word appearance (gray curve).

The autocorrelation for the word plants (panel D) shows that its usage is organized into spans extending 2000-3000 words, in agreement with the domain structure shown in panel A. A similar situation occurs in the case of instinct, for which correlation structures extend over a scale of approximately 5000 words. On the other hand, the autocorrelation for the word for falls to the random level at very short distances, thus indicating no pattern of domains in its usage.

Heterogeneity in the rate of word occurrence over the text is directly related to the specificity of that word to certain sections of the text. For instance, the use of the word instinct directly tags a few parts where, due to the specific subject being treated there, it occurs more frequently than elsewhere in the text. If the word instinct is found in the text, it is likely that that particular occurrence belongs to one of the sections in which the rate of instinct is higher. Likewise, the distribution of plants also offers hints about which part of the text the word is found in. The variability in the 

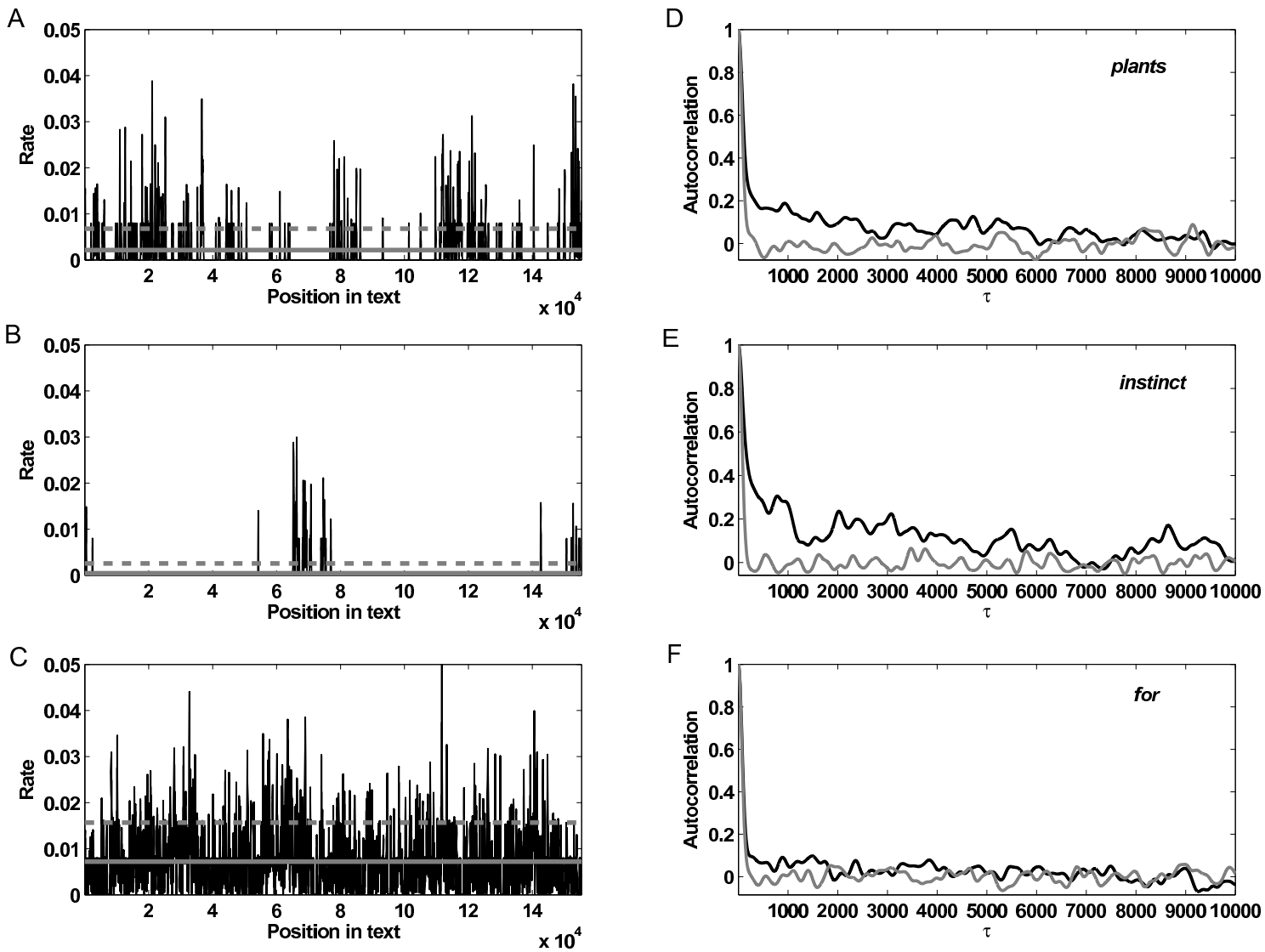

Figure 1: Distribution of three words in The Origin of Species, by Charles Darwin. Left panels (A, B, and C) show the rate of occurrence of the words plants, instinct, and for as a function of the position in the text measured in number of words (black line), its average (gray full line) and one standard deviation above the average (grey dash line). Right panels ( $\mathrm{D}, \mathrm{E}$, and $\mathrm{F}$ ) show the autocorrelation of the rate of occurrence of the same words as a function of the distance between text positions. The black line shows the autocorrelation for the word as it appears in the original text. The gray line corresponds to a random shuffling of all words in the text. 
frequency of word usage can therefore be exploited to discriminate between different sections of the text. Our aim in the following is to quantify how efficiently this discrimination can be carried out, giving a statistical measure of the amount of knowledge that the word distribution carries about the parts of the text where each word appears.

\section{Information in the distribution of words}

As discussed above, the heterogeneity in the distribution of individual words can be used to tag different sections of the text. In what follows, we consider a text divided into contiguous sections of equal length, and then define an information-theoretic measure that captures the information contributed by the distribution of all individual words in the text. We then show that this information is maximal for a characteristic size of the parts into which the text is divided -typically, of a few thousand words. We thus show that information theory can be used to characterize a new scale for linguistic structures, much longer than the range of application of grammar rules but still much shorter than, for instance, most books. As we shall see below, this scale turns out to be related to the size of semantic domains in written language.

Consider a text of $N$ words in length, and with a lexicon of $K$ different words. In our analysis, two words are considered to be different if they are spelt differently, in particular, even if they are inflected variations sharing a common root. We divide the text into $P$ parts of identical length, each part containing $s=N / P$ words. Our aim here is to define an information-theoretical measure to quantify the relationship between the heterogeneities in the distribution of words due to their linguistic function and the text partition.

We define our measure of relative information as the difference between Shannon's mutual information [7, 22, evaluated in a given text and in a surrogate version of it. The surrogate text is built form the original one by randomly shuffling all its words. In this way, the random text will have exactly the same overall word frequencies as the original text, but will lack any linguistically relevant order in the sequence of words. The basic assumption behind our definition is that the constraint of encoding linguistic information determines the degree of order by which the original text exceeds its random counterpart. In other words, our measure establishes the amount of information required to re-arrange the words of the random text to recover the word distribution over the parts of the original text. The more heterogeneous the word distribution in the original text, the more information will be required to re-order its words from the random shuffling. A direct application of Shannon's mutual information leads to the following definition (see Appendix $\mathrm{B})$ :

$$
\Delta I(s)=\sum_{w=1}^{K} p(w)[\langle\hat{H}(J \mid w)\rangle-H(J \mid w)],
$$

where the sum runs over the lexicon of the text in question, i.e. over its $K$ different words. For a word $w$ with a total of $n$ occurrences, the coefficient $p(w)=n / N$ gives its frequency over the whole 
text. The entropy-like quantity $H(J \mid w)$ is given by

$$
H(J \mid w)=-\sum_{j=1}^{P} \frac{n_{j}}{n} \log _{2} \frac{n_{j}}{n},
$$

where $n_{j}$ is the number of occurrences of word $w$ in part $j$ of the real text, and the sum runs over all the parts. Meanwhile, $\langle\hat{H}(J \mid w)\rangle$ is the same quantity calculated for a random shuffling of all the words in the text, and averaged over the all possible realizations of the random permutation of words. This average can be computed analytically (see Appendix C).

The behavior of the entropy quantities can be illustrated with an example. In Fig. 2 we show the value of the entropy for all the words appearing in The Origin of Species as a function of word frequency (black dots). We also plotted the entropy for the words in one realization of the random permutation of word positions (grey dots), and the value of the same entropy when it is averaged over an infinite number of realizations of the random text (black line). The latter corresponds to the exact analytical calculation of the entropy.

There are two important observations in regard to the comparison between the entropy of the real text and that in the random version. First, the entropy of words in the real text is on average lower than in the random version of the text for the same frequency range. The lower value of the entropy for words in the real text is a consequence of the more structured distribution of words sequences conveying complex information in contrast to the randomly located words in the shuffled text. Second, the fluctuations across different words in the same frequency range vary over a much larger range in the real text than in the shuffled one. This is due to the fact that the large variations in word distribution are not stochastic but imposed by linguistic and thematic constraints in the text. Therefore, even groups of words having the same frequency can show sharp variations in their patterns of occurrence due to their different linguistic roles.

For a specific text, the relative information defined by Eq. 2 depends only on $s$, the number of words per part, through the number of parts $P$. The variable $s$ sets a scale for the coarseness with which the distribution of words over the text is determined. At a given scale, $\Delta I(s)$ quantifies how much information about specific parts of the text is contained in the distribution of words with respect to that in the random text. Words that tend to appear evenly over the text will contribute little information about different parts. On the other hand, words with non-uniform distributions that cannot be simply associated with random fluctuations will have a significant contribution to the total relative information. As given by Eq. 2, $\Delta I(s)$ will have units of bits per word.

As an example, we describe as a function of the scale $s$ for three texts: The Origin of Species by Charles Darwin, Analysis of the Mind by Bertrand Russell, and Moby Dick by Herman Melville. The lengths of the texts were, respectively, 155800, 89586, and 218284 words. Figure 3A shows that, in all three texts, there is a scale at which the information $\Delta I(s)$ is maximal. In these examples, the maximal information values occur at a scale of approximately 3000 words for The Origin of the Species, 700 words for Analysis of the Mind and 1200 words for Moby Dick. For each text, starting from scales of around 100 words, the information encoded in the word distribution increases with s up to a specific most informative scale. This means that the distribution of words 


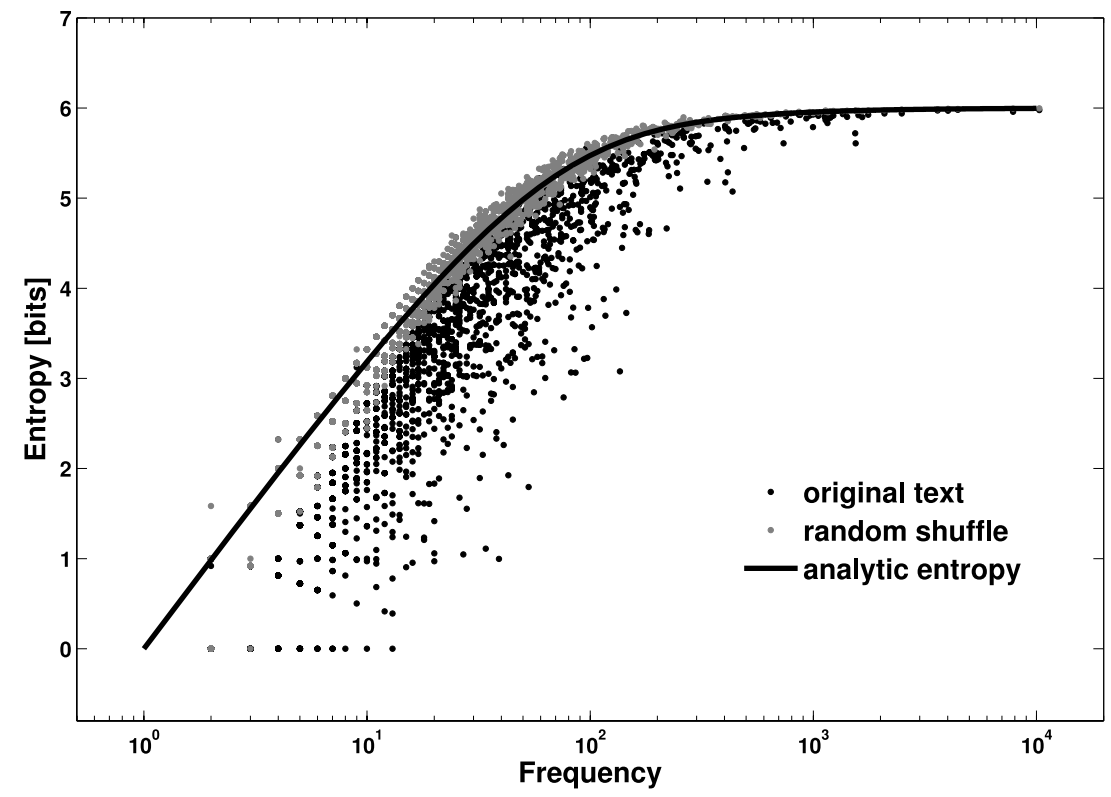

Figure 2: Word entropy for a real and a random text. The black dots show a scatter plot of the entropy in bits for all the words in The Origin of the Species as given by Eq. (3) and using a partition of the text in $P=64$ parts. The gray dots show the entropy of the words in randomly shuffled realization of the original text. The full line is the result of the analytical expression for the entropy (see Appendix C) of the words in the random version of The Origin of Species. 
better discriminates between different sections of the text for a characteristic size of the parts. It is for this particular size that the mutual information of the word distribution and the text partition differs maximally between the real and the random texts. The scales at which the information is maximal, roughly between 1000 and 3000 words in length, are much larger than the scope of grammatical rules. As we shall argue below, they are rather related to the semantic structure of the texts.

For the three texts considered above, there is no direct relation between text length and the size of the most informative scale. However, in principle, longer texts allow their semantic structures to span longer scales. To investigate this hypothesis more in detail, we analysed a corpus consisting of 5258 books in English, including English and American literature, as well as texts on science, engineering, technology, history, philosophy, and religion. All the texts were obtained from the Project Gutenberg internet site 1 . In Fig. $3 \mathrm{~B}$ we show a scatter plot of the scale at which the information $\Delta I(s)$ is maximal versus the length of the text, for all of the books in our corpus. Although the data exhibit a large dispersion, there is a clear pattern of slow growth of the most informative scale with text length. As a guide to the eye, we also plotted two straight lines with slopes 0.65 and 0.4 which, in this log-log plot, correspond to power-law functions. The most informative scale of books around 105 words in length typically lies between 300 and 3000 words. This means that, for such texts, the size of the parts at which the information $\Delta I(s)$ attains its maximum is roughly 100 times less than the text length.

It is interesting to identify which are the books that fall in extreme regions of the cloud of points in Fig. 33B. For instance, at the lower-left corner, which corresponds to short books with a small most informative scale $s$, we find works like Quotations of Lord Chesterfield, Quotes and Images From The Novels of Georg Ebers, which consist of a collection of short quotations with no thematic unity building up along the text. Consistently, the scale associated to these books lies in the range between 50 and 70 words. At the opposite extreme, at the upper-right corner of the cloud of points, which corresponds to long books and large most informative scales, we find long treatises on subjects with clear thematic unity. In particular, the three works with the largest most informative scale were History of The Decline and Fall of the Roman Empire, Vol. 3, by E. Gibbon, A History of Rome, Vol. 1, by A. Greenidge, and Civilization of the Renaissance in Italy, by J. Burckhardt.

Notwithstanding the large dispersion of the most informative scale, the maximum value of the information $\Delta I(s)$ for each book was surprisingly consistent across the corpus. Figure $3 \mathrm{C}$ shows a histogram of the maximum information for the books in the corpus. It turns out to be narrowly centred at 0.2 bits/word. This suggests that, in written English, words tag different parts of the text with very consistent accuracy, although the size of those parts may vary substantially between texts. However, when looking at the books within a given range of values of the maximum information per word we found a tendency for literary and history books to have lower values of the information per word than many of the books on science and engineering. The pattern becomes evident in Fig. 3D where we show the normalized histograms of the maximum information computed on three subsets

\footnotetext{
${ }^{1}$ www.gutenberg.org
} 

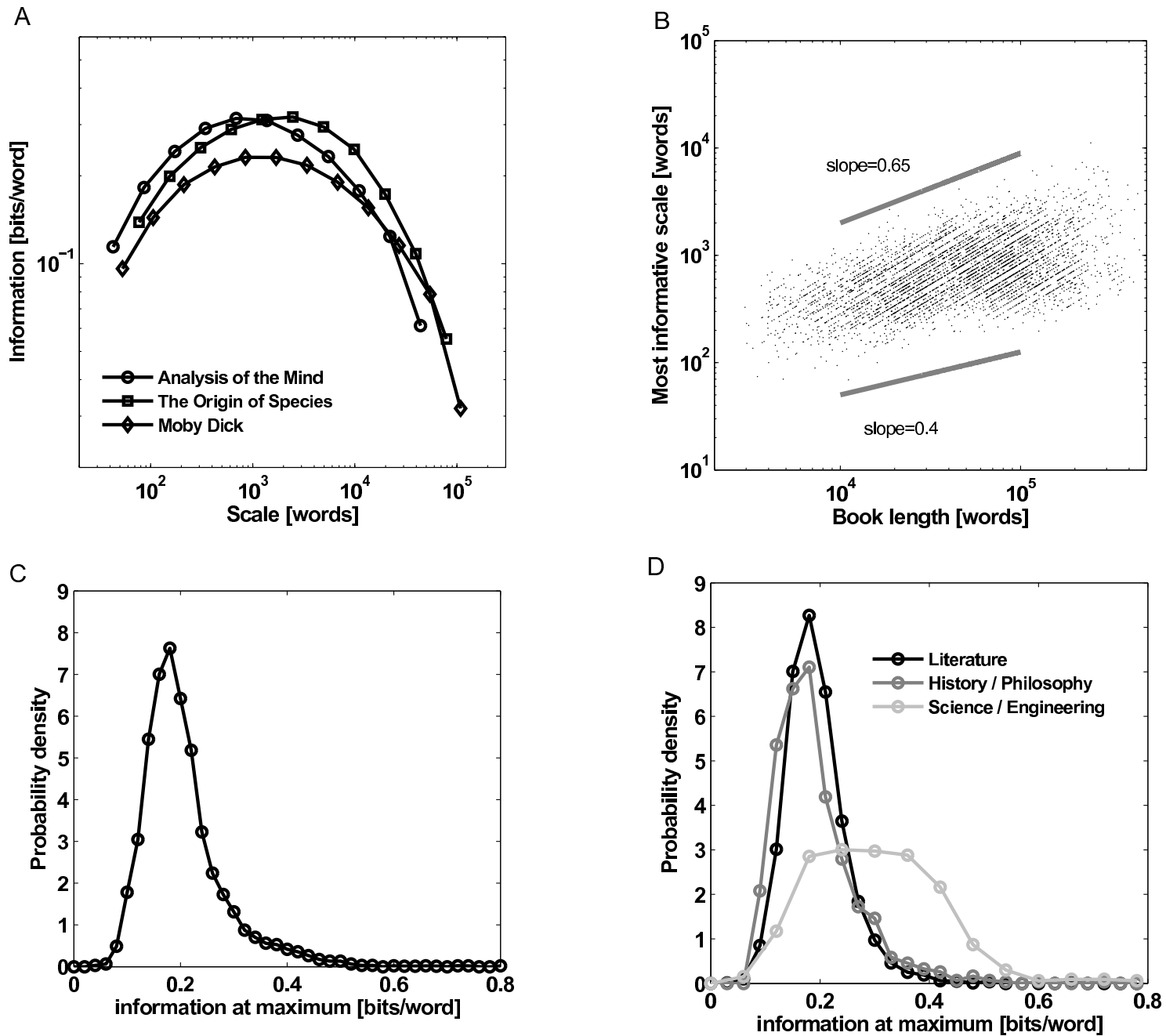

Figure 3: Information encoded in whole texts. A. Information $\Delta I(s)$ in bits per word for three texts, as a function of the scale $s$. The texts are Analysis of the Mind, by Bertrand Russell (circles); The Origin of Species, by Charles Darwin (squares); and Moby Dick, by Herman Melville (diamonds). B. Scatter plot of scale at which the total information is maximal as a function of the text length for all the texts in a corpus of 5258 books in English. The straight lines are power-law functions (note the logarithmic scales) whose slopes approximately confine the speed of growth of the most informative scale with text length. C. Normalized histogram showing the distribution of the maximum total information for all the books in the corpus. D. Normalized histogram showing the distribution of the maximum total information for each of the three partitions in which the corpus was divided. The number of books in each division of the corpus was as follows: literature, 3329 books; history and philosophy, 1374 books; science and engineering, 555 books. 
of the whole corpus. Whereas the information for the literary, and history and philosophy books has a very similar distribution characterized by a narrow peak around 0.2 bits/word, the values for science and engineering books have a broader distribution that extends over higher values of the information. This shows that $\Delta I(s)$ can quantify the similarities and difference in language styles in the different components of the corpus. In particular, it indicates that the usage of language in scientific and engineering books is such that the distribution of words tags more efficiently the different parts of the text.

\section{The information of individual words}

We deal, in this section, with the contribution of individual words to the information $\Delta I(s)$. It turns out that each word has its own characteristic scale, at which the information it provides about specific parts of the text is maximal. By means of a heuristic model, we show that the existence of that scale is a consequence of the domain structure of word distribution.

Equation 2 shows that $\Delta I(s)$ is additive over the whole lexicon. Thus, each term in the sum can be interpreted as the contribution of a single word to the total information. For a word $w$, its contribution to the total information equals $\Delta I_{w}(s)=p(w)[\langle\hat{H}(J \mid w)\rangle-H(J \mid w)]$. This means that we can not only compute the overall information as a function of the scale $s$, but also associate a measure of information with every different word appearing in the text. Thus, by using the single-word information $\Delta I_{w}(s)$, it is possible to identify the most informative words in a given text. Remarkably, as we shall illustrate below for The Origin of Species, the most informative words coincide with those words that any human reader would choose as most representative of the subject of the text. Therefore, summing the contributions of every word, the total information $\Delta I(s)$ can be interpreted as a measure of the overall semantic information in the text in question.

The mathematical form of $\Delta I_{w}(s)$ also suggests another interpretation of the meaning of the overall information $\Delta I(s)$. The contribution of each word to the total information is proportional to both the frequency of the word and to the difference in entropy of the word distributions in the random and real texts. The difference in entropies is a direct measure of the degree of order that exists in the word distribution in the real text beyond that determined by the word frequencies alone. Ultimately, that order has an origin in the semantic role of the word. That means that for a word of a given frequency $p(w)$, the more heterogeneous the distribution of the word compared to its stochastic counterpart in the random text, the larger its contribution to the total information.

To capture the role of individual words in determining the semantic information of the text, we analysed the information encoded in single words as a function of the scale $s$. In Fig. 4 we show the results obtained for The Origin of Species.

Symbols in Fig. 4A stand for the single-word information $\Delta I_{w}(s)$ as a function of $s$ for the words plants, instinct, and for. The profile of $\Delta I_{w}(s)$ vs. $s$ is qualitatively the same for the three words, with a maximum at an intermediate scale. The maximum is located at 1216 for plants, at 4864 for instinct, and at 76 for the word for. Thus, the most informative scale is different for each word, but roughly lies in the interval where the total information $\Delta I(s)$ of different texts attains 

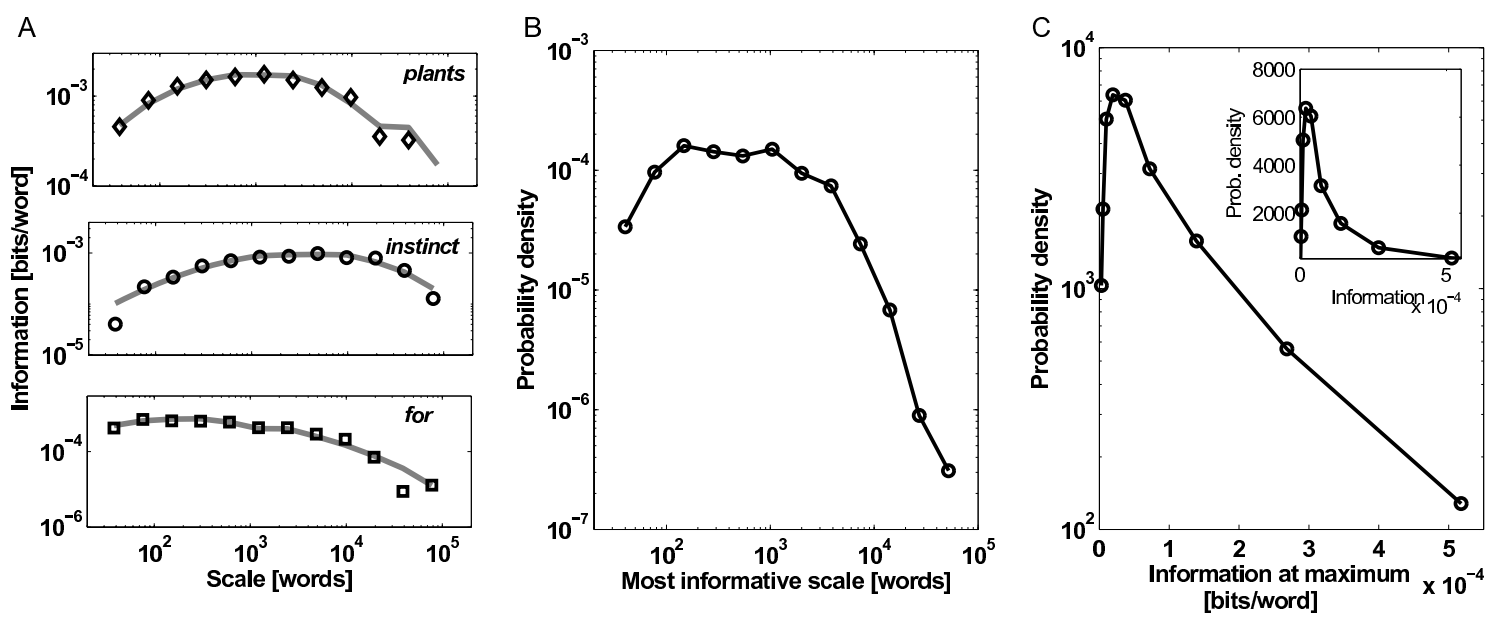

Figure 4: Information encoded in single words. A. The three subpanels show the information of single words for the three examples shown in Fig. 1, as a function of the scale $s$. The full lines are fittings obtained using the model described in the text. B. Probability density of the most informative scale for all the words in the The Origin of the Species. C. Probability density of the maximum information for all the words in The Origin of the Species.

its maximum (see panels A and B in Fig. 3). We show below that this scale can be related to the size of typical domains in the distribution of each word. The most informative scale of a whole text is ultimately determined by the interplay between the positions of the maxima of its individual words.

In Fig. $4 \mathrm{~B}$ we have plotted a normalized histogram of the most informative scale for all the words in the book. It exhibits a flat maximum spanning scales between 100 and 1000 words approximately, after which it decays sharply. In Fig. $4 \mathrm{C}$ we show a histogram of the value of the maximum information for the individual words. As it was the case in Fig. $3 \mathrm{C}$, the distribution is narrowly cantered around a typical information value of about bits/word.

The behavior of the single-word information as a function of the scale $s$ can be heuristically explained by means of a simple model. It consists of a stochastic representation of the distribution of the $n$ occurrences of a given word over the text of length $N$. Our main assumption is that the word in question has a uniform random distribution all over the text, except for a localized domain where its frequency is larger than in the rest. The distribution is specified by giving the length $L$ of the domain where the word is more concentrated, the place $N_{0}$ in the text at which the domain begins, and the excess ne of occurrences of the word in the domain.

Once the three parameters $L, N_{0}$, and $n_{e}$ have been specified, the distribution is built by allocating the $n$ occurrences of the word along the text, following two steps: (i) A number $n-n_{e}$ of occurrences are uniformly distributed at random all over the text. (ii) The remaining ne occurrences are uniformly distributed at random over the concentration domain, i.e. between places $N_{0}$ and 
$N_{0}+L-1$. Of course, only one occurrence per place is allowed. Also, the domain length $L$ is supposed to be large enough as to allow for the ne excess occurrences once step (i) is completed. The expected number of occurrences inside the domain is $n_{e}+(n-n e) L / N$ while, in the remaining of the text, any portion of length $L$ contains, on the average, just $\left(n-n_{e}\right) L / N$ occurrences. The random distribution is build numerically, and the value of $\Delta I_{w}(s)$ as a function of the number of words per part is averaged over realizations of the allocation process.

The effect of varying any of the three parameters $L, N_{0}$, and $n_{e}$, leaving the other two fixed, can be qualitatively described as follows. The domain length $L$ controls the position of the maximum of $\Delta I_{w}(s)$, which roughly coincides with $L$. Simultaneously, since as $L$ grows the difference in frequency between the domain and the remaining of the text decreases, larger values of $L$ imply smaller values of $\Delta I_{w}(s)$. Conversely, a growth in the excess ne increases the difference in frequency, and therefore implies a growth in $\Delta I_{w}(s)$. Finally, changes of the domain position upon variation of $N_{0}$ have practically no effect on the position and height of the maximum of $\Delta I_{w}(s)$, but control the form in which $\Delta I_{w}(s)$ decays at both sides of the maximum.

The grey curves in the three sub-panels of Fig. 4A show the single-word information for plants, instinct, and for, estimated using our model. The agreement is excellent, strongly suggesting that the domain-like distribution pattern of words along the text is the most relevant factor determining the characteristic behavior of $\Delta I_{w}(s)$.

Finally, we provide evidence that the information captured by $\Delta I_{w}(s)$ is related to the semantic role of words. With this aim, we computed the single-word information for all the words in The Origin of the Species, in Analysis of the Mind, and in Moby Dick, at the scale $s$ where the total information $\Delta I(s)$ was a maximum for each of the texts (see Fig. $3 \mathrm{~A} \mathrm{~A}$ ). In Table 1, we present the first words of the three texts ranked by the information $\Delta I_{w}(s)$ encoded by each word. Note, from Eq.refDI, that the contribution of each word to the total information is weighted by its frequency in the whole text, $p(w)$. Nonetheless, very few of the most frequent words -such as the, and, of, or- appear at the top of the lists. This is because, in real texts, the most common words have distributions which do not differ much from that of a typical realization of the randomly shuffled text. Remarkably, on the other hand, the words with largest information are specifically relevant to the main subjects of the text. Among the top ten words of The Origin of Species, for instance, we find species, varieties, hybrids, forms, islands, selection and genera. Those words would certainly be signalled out by a reader as some of the most representative of the message conveyed by the text. In Analysis of the Mind we recognize a very similar situation. Its top words are essential to the philosophical subject of the book. The comparison with Moby Dick is interesting because, it being a novel, its style is very different to that of Darwin's and Russell's treatises. As in other novels, much of the structure is built up around its characters, through a network of relationships that change throughout the text. This is evidenced in the list of Table 1 by the prominence of pronouns and proper names, in addition to the nouns that set the thematic focus of the book. A small number of words in the example listings have been assigned a large value of information without being representative of the main subject of the texts. That is likely due to fluctuations in the distributions of those words, that affect the estimation of their information. We have obtained listings like those of Table 1 for a variety of texts in different languages, with highly consistent 
Table 1: Most informative words for three books.

\begin{tabular}{|c|c|c|}
\hline The Origin of Species & Analysis of the Mind & Moby Dick \\
\hline on & image & I \\
species & memory & whale \\
varieties & images & you \\
hybrids & word & Ahab \\
forms & belief & is \\
islands & words & ye \\
of & desire & Queequeg \\
will & sensations & thou \\
selection & object & me \\
genera & you & of \\
plants & past & he \\
seeds & knowledge & captain \\
sterility & box & boat \\
fertility & content & the \\
characters & consciousness & Stubb \\
breeds & appearances & his \\
groups & movements & Jonah \\
water & mnemic & was \\
the & feeling & whales \\
formations & proposition & my \\
pollen & general & him \\
bees & particulars & Starbuck \\
instincts & thought & sir \\
new & experience & white \\
he & objective & sperm \\
rudimentary & meaning & bildad \\
cells & laws & her \\
organs & introspection & we \\
intermediate & animal & Peleg \\
crossed & vague & said \\
natural & sensation & fish \\
birds & physical & Pip \\
would & habit & old \\
I & matter & a \\
domestic & cook \\
wax & flask \\
forgan & aye \\
\hline
\end{tabular}


results. In all cases, the words with the largest values of $\Delta I_{w}(s)$ were among the most relevant to the respective subjects. It is remarkable that a simple measure based on Shannon mutual information can readily identify the most important words in individual texts.

\section{Discussion}

The richness and diversity of linguistic structures pose a challenge to the characterization of the complex information conveyed by language using the quantitative techniques. Here we used a direct application of information theory to quantify semantic information in language sequences. Our main hypothesis was that the key signatures of the semantic content of a text will be reflected in long-range statistical patterns in the use of words, and thus should be captured by basic information theoretic quantities. In particular, we introduced a measure of relative information, $\Delta I(s)$, that gauges a degree of order in written language based on the statistical description of word frequencies in sections of the text of a given size $s$.

For individual texts, the information $\Delta I(s)$ characterizes the presence of an optimal scale $s$ at which the information attains a maximum. If a text is divided into sections of the size of the optimal scale, the distribution of words in those parts will be statistically the most diverse. For single texts with an overall thematic unity, those divisions represent the typical spans in which lines of argument and semantic structure develop. An empirical support for this interpretation comes from the inspection of specific written works. Books with the smallest optimal scales were those made up of collections of disparate short text fragments. On the contrary, the books with the largest optimal scales were long treatises on well defined subjects. For texts such as Darwin's The Origin of Species, Russell's Analysis of the Mind, and Melville's Moby Dick, the optimal scales were in the range between 1000 and 3000 words. This is roughly the length needed to develop a line of argument in a literary or scholarly text. While it can probably be natural to expect that complex messages in written language build up on scales of a few thousands words, to the extent of our knowledge, we proposed the first information-theoretic quantification of those long range semantic structures.

The information $\Delta I(s)$ is built up from the contributions of the information associated with individual words. The study of the information per word, $\Delta I_{w}(s)$, revealed the presence of a maximum at a scale that depended on the particular word. That optimal scale is such that the distribution of the word over the text partition differs the most from that of a word with the same frequency but otherwise randomly distributed over the text. Using a simple model of word distribution we showed that the optimal scale for single words is related to the size of the typical domains, or clusters, in which words tend to appear in written language.

Moreover, when words are ranked according to their information contribution, the most informative ones are those clearly representative of the message in the text. Remarkably, these most informative words could be identified by using our information-theoretical measure without any a priori knowledge of the language or the text, apart from the identification of the elementary information-carrying tokens words themselves. 
Probably, similar features can be found in other information carriers in nature, where a particular kind of semantic information leaves its mark on the long-range distribution of the basic symbols. Much of the insight gained in our study could thus be extended to the analysis of other biological information structures, like some neural signals, the genetic code, and patterns of animal communication and behavior. Overall, our results suggest that complex aspects of the information encoded in symbolic sequences are susceptible of quantitative characterisation and analysis using the rigorous principles of information theory.

\section{Acknowledgements}

We are grateful to Inés Samengo for her critical reading of the manuscript. This work was supported by the UK Medical Research Council and the Royal Society (MAM), and CONICET and ANPCyT, Argentina (DHZ).

\section{References}

[1] Alvarez-Lacalle, E., Dorow, B., Eckmann, J. P., and Moses, E., Hierarchical structures induce long-range dynamical correlations in written texts, Proc Natl Acad Sci U.S.A 103 (2006) $7956-7961$.

[2] Arfken, G. B. and Weber, H.-J., Mathematical methods for physicists, 6th edn. (Elsevier, Boston, 2005).

[3] Bray, A. J., Theory of phase-ordering kinetics, Advances in Physics 43 (1994) 357-458.

[4] Cancho, R. F. i. and Sole, R., Two regimes in the frequency of words and the origins of complex lexicons: Zipf's law revisited, Journal of Quantitative Linguistics 8 (2001) 165-173.

[5] Cancho, R. F. i. and Sole, R. V., The small world of human language, P Roy Soc B-Biol Sci 268 (2001) 2261-2265.

[6] Cancho, R. F. i. and Sole, R. V., Least effort and the origins of scaling in human language, Proc Natl Acad Sci U.S.A 100 (2003) 788-791.

[7] Cover, T. M. and Thomas, J. A., Elements of information theory, 2nd edn. (J. Wiley, Hoboken, N.J., 2006).

[8] Deacon, T. W., The symbolic species : the co-evolution of language and the brain, 1st edn. (W.W. Norton, New York, 1997).

[9] Dorogovtsev, S. N. and Mendes, J. F., Language as an evolving word web, P Roy Soc B-Biol Sci 268 (2001) 2603-6. 
[10] Ebeling, W. and Poschel, T., Entropy and long-range correlations in literary english, Europhysics Letters 26 (1994) 241-246.

[11] Feller, W., An introduction to probability theory and its applications, Wiley series in probability and mathematical statistics, 3rd edn. (Wiley, New York, 1968).

[12] Herrera, J. P. and Pury, P. A., Statistical keyword detection in literary corpora, European Physical Journal B 63 (2008) 135-146.

[13] Kintsch, W. and van Dijk, T. A., Toward a model of text comprehension and production, Psychological Review 85 (1978) 363-394.

[14] Lieberman, E., Michel, J. B., Jackson, J., Tang, T., and Nowak, M. A., Quantifying the evolutionary dynamics of language, Nature 449 (2007) 713-716.

[15] Maynard Smith, J. and Szathmáry, E., The origins of life : from the birth of life to the origin of language (Oxford University Press, Oxford ; New York, 1999).

[16] Montemurro, M. A. and Pury, P. A., Long-range fractal correlations in literary corpora, Fractals 10 (2002) 451-461.

[17] Montemurro, M. A. and Zanette, D. H., Entropic analysis of the role of words in literary english, Advances in Complex Systems 5 (2002) 7-17.

[18] Nowak, M. A., Komarova, N. L., and Niyogi, P., Computational and evolutionary aspects of language, Nature 417 (2002) 611-617.

[19] Nowak, M. A., Plotkin, J. B., and Jansen, V. A. A., The evolution of syntactic communication, Nature 404 (2000) 495-498.

[20] Ortuño, M., Carpena, P., Bernaola-Galvan, P., Munoz, E., and Somoza, A. M., Keyword detection in natural languages and dna, Europhysics Letters 57 (2002) 759-764.

[21] Schurmann, T. and Grassberger, P., Entropy estimation of symbol sequences, Chaos 6 (1996) $414-427$.

[22] Shannon, C. E., A mathematical theory of communication, Bell System Technical Journal 27 (1948) 379-423.

[23] Shannon, C. E., Prediction and entropy of printed english, Bell System Technical Journal 30 (1951) 50-64.

[24] Sigman, M. and Cecchi, G. A., Global organization of the wordnet lexicon, Proc Natl Acad Sci US A 99 (2002) 1742-7.

[25] Zanette, D. H. and Montemurro, M. A., Dynamics of text generation with realistic zipf's distribution, Journal of Quantitative Linguistics 12 (2005) 29-40. 


\section{Appendix A. Rate of word occurrence}

In this section we give details about the calculation of the rate of word occurrence shown in Fig. 1 of the main text.

For a text of length $N$, we denote the positions of successive words by an index $t=1, \ldots, N$. The positions of a particular word $w$, that appears $n$ times in the text, are then represented by variables $t_{i}$, with $i=1, \ldots, n$. Thus, the density of occurrences of word $w$ can be represented as

$$
\nu_{w}(t)=\sum_{j=1} \delta\left(t-t_{j}\right)
$$

where $\delta(t)$ is the Dirac delta function [2]. In order to reveal the domain-like patterns in the distribution of words, we define a rate of occurrence for each word as a convolution of the density $\nu_{w}(t)$ with a bell-shaped kernel,

$$
\rho_{w}(t)=\int_{-\infty}^{\infty} G\left(t-t_{w}^{\prime}, \sigma\right) \nu_{w}\left(t^{\prime}\right) d t^{\prime}
$$

where for simplicity of notation we assumed that the variable $t$ is continuous. In particular, we used the following zero-mean Gaussian kernel:

$$
G(t, \sigma)=\frac{e^{-\frac{t^{2}}{2 \sigma^{2}}}}{\sqrt{2 \pi} \sigma},
$$

where the parameter $\sigma$ controls the width. It can be shown that this type of kernel introduces correlations in the rate $\rho_{w}(t)$ over spans of order of $\sigma$. To obtain the data for Fig. 1 we used $\sigma=50$ words. Therefore, all the structure show in the figure can be attributed to genuine correlations in the distributions of words.

\section{Appendix B. Derivation of $\Delta I(s)$}

In this section we supply additional details on the derivation of the information measure $\Delta I(s)$, given by Eq.2,

Let us consider a given word $w$, which appears $n_{j}$ times in part $j$, with $j=1, \ldots, P$. We can then define the conditional probability of finding word $w$ in part $j$, as $p(w \mid j)=n_{j} / N_{j}$. If $K$ is the size of the lexicon, the normalization condition for the above probability is $\sum_{w=1}^{K} p(w \mid j)=1$. If we now call $p(j)=N_{j} / N$ the a priori probability that the word $w$ appears in part $j$, then $\sum_{j=1}^{P} p(w \mid j) p(j)=p(w)$, where $p(w)=n / N$ stands for the overall probability of occurrence of a word in the whole text. 
The probability $p(w \mid j)$ tells us how likely is to find word $w$ given that we are looking into part $j$. Here we are interested in assessing how well a particular word tags a section, or part, in a text. Such information is given by the inverted probability $p(j \mid w)$, which tells how likely is that we are looking into part $j$ given that we saw an instance of word $w$ in the text. This probability is easily found by means of Bayes's rule [11], as follows:

$$
p(j \mid w)=\frac{p(w \mid j) p(j)}{\sum_{i=1}^{P} p(w \mid i) p(i)} .
$$

Then, by writing explicitly the probabilities in the right-hand side of Eq. (7), we find that $p(j \mid w)=$ $n_{j} / n$.

With the previous definitions we can write Shannon mutual information between the text sections and the words [7]:

$$
M(J, W)=\sum_{w=1}^{K} p(w) \sum_{j=1}^{P} p(j \mid w) \log _{2}\left(\frac{p(j \mid w)}{p(j)}\right) .
$$

However, as was discussed in the main text, we are interested in a measure that quantifies the specific linguistic information in the long-range distribution of words. We therefore need to subtract the residual information contributed by fluctuations in word frequencies over the different parts. These fluctuations are particularly important for words that occur a number of timesn $<<$ [17, 21].

Let us call $\hat{M}(J, W)$ the mutual information computed on one particular random realization of the text obtained by shuffling all of the words' positions. In order to obtain a representative quantity that does not depend on one particular realization of the random shuffling, we take an average over all the possible realizations of the random text, represented as $\langle\hat{M}(J, W)\rangle$. We can now define the information in the distribution of words simply as the difference in the value of the mutual information calculated on the real and the average over the random texts, $\Delta(s)=$ $M(J, W)-\langle\hat{M}(J, W)\rangle$, where we made explicit the dependency on the scale parameter $s$. After expanding and regrouping terms we can write the relative information $\Delta I(s)$ as follows:

$$
\Delta I(s)=\sum_{w=1}^{K} p(w)[\langle\hat{H}(J \mid w)\rangle-H(J \mid w)],
$$

The first of the entropies appearing in Eq. (9) corresponds to the one computed on the random text and averaged over all the realizations of the random permutation of words, and is defined as follows:

$$
\langle\hat{H}(J \mid w)\rangle=-\sum_{j=1}^{P}\left\langle\hat{p}(j \mid w) \log _{2} \hat{p}(j \mid w)\right\rangle,
$$

where we denote by $\hat{p}(j \mid w)$ the probabilities computed on the random text. The second entropy is estimated directly on the real text, and its definition is the following:

$$
H(J \mid w)=-\sum_{j=1}^{P} p(j \mid w) \log _{2} p(j \mid w) .
$$


In our approach, the amount of information contributed by single words to the total information is proportional to the difference in entropies for the word in the random version of text and the one on the real text. The proportionality factor is given by the a weight equal to the overall probability of a word in the text, $p(w)$.

\section{Appendix C. Analytic computation of the entropy of the random text}

Here we compute an analytic expression for the entropy $\langle\hat{H}(J \mid w)\rangle$, appearing in Eq. (9). As was discussed above this entropy is computed on a stochastic version of the text obtained by randomly shuffling all the words' positions. The brackets $\langle\ldots\rangle$ denote an average over all possible randomly shuffled texts.

Again, let us suppose that the random text has a length of $N$ words, and that it is divided in $P$ parts of equal length. Using Eq. (10), for a word that appears $m_{j}$ times in part $j$ with a frequency $n$ over the whole text, this entropy takes the following form:

$$
\hat{H}(J \mid w)=-\sum_{j=1}^{P} \frac{m_{j}}{n} \log _{2} \frac{m_{j}}{n} .
$$

The average of the entropy $\hat{H}(J \mid w)$ over all possible realizations of the random text is computed as follows:

$$
\langle\hat{H}(J \mid w)\rangle=-\sum_{\substack{m_{1}+\ldots+m_{P}=n \\ \text { with } m_{j} \leq N / P, j=1, \ldots, P}} p\left(m_{1}, \ldots, m_{P}\right) \sum_{j=1}^{P} \frac{m_{j}}{n} \log _{2} \frac{m_{j}}{n},
$$

where $p\left(m_{1}, \ldots, m_{P}\right)$ is the probability of finding $m_{j}$ words in part $j$, with $j=1, \ldots, P$. We notice that in Eq. (13), for each of the $P$ terms in the second sum, the first sum can be taken over all indices except one. This leads to the following simpler form of the average entropy:

$$
\langle\hat{H}(J \mid w)\rangle=-P \sum_{m=1}^{\min \{n, N / P\}} p(m) \frac{m}{n} \log _{2} \frac{m}{n} .
$$

Finally, the marginal probability $p(m)$ can now be easily computed. It is given by the probability of finding $m$ instances of word $w$ in one part, together with $N / P-m$ words different from $w$, and reads

$$
p(m)=\frac{\left(\begin{array}{c}
n \\
m
\end{array}\right)\left(\begin{array}{c}
N-n \\
N / P-m
\end{array}\right)}{\left(\begin{array}{c}
N \\
N / P
\end{array}\right)} .
$$

